\title{
Automatic Control of Dissolved Oxygen Concentration in Stirred Microbial Cultures
}

\author{
By D. G. MACLENNAN AND S. J. PIRT \\ Microbiology Department, Queen Elizabeth College, London, W. 8 \\ (Received 14 February 1966)
}

\section{SUMMARY}

Equipment was developed for the automatic control of dissolved oxygen concentration in microbial cultures, by using the Mackereth electrode as a sensing probe. Factors affecting the choice of components are discussed and the performance of the equipment is described during typical batch and continuous culture runs. The equipment provided precise and reliable control over a wide range of dissolved oxygen concentrations, and retained a high degree of stability over long periods of continuous use.

\section{INTRODUCTION}

Ever since the inception of aerobic techniques for cultivating micro-organisms there has been a need for quantitatively assessing the concentration of dissolved oxygen in the culture medium. Until relatively recently, the main concern was to ensure that growth was not impaired by an inadequate supply of oxygen. Over the last decade, however, there has been an increasing interest in the response of microorganisms to conditions of limited oxygen availability. Thus the necessity arose not only for the direct measurement of dissolved oxygen in microbial cultures, but also for the automatic control of this factor.

The implementation of automatic control of dissolved oxygen in culture media has been considerably delayed by the lack of a suitable measuring probe. Such a probe must remain stable over long periods of time without requiring recalibration, must have a linear response to increasing oxygen concentration and must be capable of sterilization. The requirement for stability becomes even more rigorous when the electrode is to be used in continuous processes, which may be required to run for periods of a month or more. It is in long-term stability that most electrodes described to date have proved deficient. An electrode suitable for use with automatic control equipment, however, has recently been described by Mackereth (1964), and a smaller version suitable for laboratory use is now commercially available (Electronic Instruments Ltd., Richmond, Surrey).

Herbert, Phipps \& Tempest (1965) briefly described an 'oxystat' for controlling the dissolved oxygen concentration in continuous microbial cultures, by using the Mackereth electrode. The authors stated that reasonably good control was achieved over a wide range of dissolved oxygen concentrations; however, few details of the control equipment and none of the performance were given. Siegell \& Gaden (1962) also described equipment for the automatic control of dissolved oxygen concentrations in fermentations; however, again, the performance of the equipment was not treated in detail and results were given only for short batch cultures (about 
$8 \mathrm{hr}$ ) at relatively high dissolved oxygen concentrations (equivalent partial pressure, 0.1 atmosphere). Lengyel \& Nyiri (1965) described an automatic aeration system based on measurement of the redox potential of the culture. No experimental results were presented, however, about the calibration of the electrode, or the accuracy and stability of the system. Furthermore, the nature of the relationship between the redox potential and the dissolved oxygen concentration is not clear. The present paper describes the design and performance of equipment for the accurate control of dissolved oxygen concentration in batch cultures and in continuous cultures of micro-organisms.

\section{METHODS}

Units. The oxygen content of a gas or liquid can be expressed either as oxygen concentration or as oxygen partial pressure. The partial pressure of oxygen in a liquid is equal to the partial pressure of oxygen in a gas in equilibrium with that liquid, and is related directly to the oxygen concentration as follows:

$$
\boldsymbol{P}=f C,
$$

where $\boldsymbol{P}$ is the partial pressure or tension of oxygen in the liquid, $C$ is the oxygen concentration in the liquid and $f$ is an activity coefficient. It is important to note that the value of $f$ depends on the nature of the liquid and thus may vary with different media or even with different cultural conditions. Membrane electrodes such as the Mackereth electrode measure the partial pressure of oxygen rather than the concentration (Siegell \& Gaden, 1962). The term 'dissolved oxygen tension' will be used throughout this work to denote the partial pressure of oxygen in the liquid phase and will be expressed as $\mathrm{mm}$. $\mathrm{Hg}$. The differences between oxygen concentration and oxygen tension have been further discussed by Kinsey \& Bottomley (1963).

\section{Characteristics of the Mackereth oxygen electrode}

The Mackereth electrode is a lead-silver galvanic cell sheathed in a protecting membrane. The cell generates a current which is directly proportional to the rate of diffusion of oxygen through the membrane, and hence to the dissolved oxygen tension in the surrounding medium. The membrane is necessary to prevent poisoning of the electrode; however, it is responsible for a time lag between a change of dissolved oxygen tension in the culture and the response of the electrode. Mackereth (1964) estimated the lag at $17 \cdot 7^{\circ}$ to be about $15 \mathrm{sec}$. for the electrode to register $90 \%$ of a change, and about 90 sec. for a $100 \%$ response. Such a lag in the electrode might introduce some difficulty in automatic control.

Besides long-term stability, perhaps the greatest advantage of the Mackereth electrode is the high sensitivity (electrode current/dissolved oxygen tension). Sheathed with the standard polythene membrane (thickness 0.0025 in.), the electrode produces approximately $2.5 \mu \mathrm{A} / \mathrm{mm}$. $\mathrm{Hg}, \mathrm{O}_{2}$, in water at $31^{\circ}$; with a fluoroethylenepropylene (FEP) membrane (thickness 0.001 in.) the output is increased to about $5.0 \mu \mathrm{A} / \mathrm{mm}$. $\mathrm{Hg}, \mathrm{O}_{2}$ (MacLennan \& Pirt, 1965). Such currents can be easily measured directly without need for pre-amplification, by measuring the potential drop across a resistor on a self-balancing potentiometric recorder. 
Mackereth (1964) found that the response time of the electrode decreased with decreasing resistance in the external circuit. Thus it was desirable to keep this resistance as low as possible, commensurate with the production of an accurately measurable potential drop across it. A full-scale deflexion of $0-1 \mathrm{mV}$ is about the lowest measuring range readily available in industrial grade self-balancing potentiometric recorders. For the polythene sheathed electrode (output about $400 \mu \mathrm{A}$ at air saturation and $30^{\circ}$ ) a resistance of about 2.5 ohms would produce such a potential drop, and minimize lag in response.

An extremely important factor influencing the performance of the electrode is the necessity for sufficient turbulence in the culture to ensure that the rate of diffusion of oxygen through the membrane is always the limiting factor. This is essential, since the output from the electrode is directly proportional to this rate of diffusion (Mackereth, 1964). In vigorously stirred fermentor vessels, however, this should not present any problem.

Another desirable characteristic of the electrode is the low residual current at zero oxygen tension ( $1 \%$ of air saturation current or better).

\section{Process characteristics}

The first factor to be considered in relation to the process was the method by which the dissolved oxygen tension in the culture was to be increased or decreased. The rate of solution of oxygen from a gas into culture medium can be described by the following equation (Arnold \& Steel, 1958):

$$
N_{A}=K_{g} \cdot a\left(P_{g}-P_{e}\right),
$$

where: $N_{A}$ is the rate of oxygen transfer; $K_{g}$ is the overall oxygen transfer coefficient expressed in terms of a partial pressure driving foree; $a$ is the total interfacial area, generally included with the overall mass transfer coefficient as $K_{g} . a$; $\boldsymbol{P}_{g}$ is the partial pressure of oxygen in the gas; $\boldsymbol{P}_{e}$ is the dissolved oxygen tension in the culture medium.

When it is necessary for the controller to change the rate of oxygen transfer $N_{A}$, in order to maintain the dissolved oxygen tension in the culture $\boldsymbol{P}_{e}$ at a predetermined value, then from the above equation the change must be brought about by altering either the mass transfer factor $K_{g} . a$, or the partial pressure of oxygen in the gas $\boldsymbol{P}_{g}$. In a stirred fermentor $\boldsymbol{K}_{g} . a$ can be varied within limits by changing the stirring rate, and $\boldsymbol{P}_{g}$ can be varied by changing the oxygen content of the inflowing gas. Although both methods are feasible for controlling dissolved oxygen tension, each method has certain advantages. Control by stirring, as used by Herbert et al. (1965), requires only one gas, usually air, whereas the partial pressure method may also require an inert carrier gas such as nitrogen. The stirring method, however, could not be applied to larger-scale equipment because of the extreme difficulty in quickly changing the speeds of large and highly powered motors. Another disadvantage of the stirring method is that the mixing efficiency might vary enormously. As the immediate use for the automatic control equipment was to study the growth of bacteria on hydrocarbon substrates, mixing was a vital factor and hence control by variation of oxygen partial pressure became essential. Such a control could be achieved by automatically controlling the adjustment of a valve which governed the flow of an oxygen-containing gas into a constant flow of an inert carrier gas. 
Before the automatic control equipment could be selected, however, there were two basic characteristics of the process which had to be taken into account: load changes and process lag.

A change in the dissolved oxygen tension due to altered conditions in the process constitutes a load change. Both the magnitude and the rate of load change must be considered. Such changes may result from changes in the rate of oxygen uptake by the culture. In batch cultures, for example, the oxygen uptake rate increases enormously through the growth period, although these changes do not occur very rapidly. An example of relatively large and rapid load changes is the fluctuation in dissolved oxygen tension observed during the 'steady state' continuous culture of Klebsiella aerogenes at 'critical' dissolved oxygen tensions (Harrison \& Pirt, 1965). Load changes could also result from a variation in the flow rate or composition of the aerating gas, or from periodic addition of antifoam to the culture. In the former case the changes would probably be relatively small and slow, but the addition of antifoam might cause large and rapid changes in dissolved oxygen tension in the culture.

The process lag is the time required for the dissolved oxygen tension to reach a new value when load changes occur. This lag is considerably influenced by the oxygen capacitance of the process, which is defined as the quantity of oxygen necessary to raise the dissolved oxygen tension in the process by $1 \mathrm{~mm} . \mathrm{Hg}, \mathrm{O}_{2}$. Although a low process capacitance is desirable in decreasing the process lag, too low a 'demand side' capacitance (that of the medium) can be a disadvantage in that the system is extremely sensitive and cannot absorb even small load changes without reflecting considerable change in the dissolved oxygen tension. This effect becomes even more troublesome when the 'supply side' capacitance (that of the gas volume above the medium in a vortex-aerated fermentor) is appreciable, because after corrective action by the controller has ceased, oxygen will continue to be transferred to the culture until the oxygen tensions in the gas phase and in the medium are the same. If the supply side capacitance is high, considerable overshoot will result. In the vortex-aerated fermentor used in the present work the supply side capacitance was appreciable, and since the demand side capacitance was low, some difficulty was expected with overshoot.

\section{Selection of controller}

Summarizing the relevant characteristics of the system, the electrode might have a considerable lag, and under some conditions the process might exhibit large and rapid load changes. In addition, the process lag might be significant and the oxygen capacitance of the system might give rise to considerable overshoot. For such a system the simplest mode of control, two position, would not be suitable, since it would give rise to excessive cycling. A more refined method involving proportional action would thus be necessary. The proportional controller moves the control valve proportionally to the deviation from the set-point. It moves the valve to a fixed position for each value of dissolved oxygen tension in the proportional band. However, when a load change occurs, a different valve position to that initially fixed will be required to maintain the dissolved oxygen tension at the set-point. As the proportional controller cannot change the fixed relationship between the valve position and the dissolved oxygen tension it will begin to control about a new value, 
thus producing 'offset'. The introduction of automatic 'reset' action effectively eliminates offset by shifting the proportional band about the set-point.

Although proportional + reset control may be satisfactory, the lags in both the electrode and the process might cause difficulty, particularly during fast load changes. Thus, in addition, 'rate action' was considered to be desirable. Rate action applies a correction to the movement of the control valve according to the rate of change of the controlled variable. It greatly speeds return to the set-point by instigating a large initial over-correction, then begins to remove this effect, leaving only the proportional + reset action to determine the final position of the valve. A treatise covering all relevant aspects of automatic process control is given by Porter \& Considine (1950), but the technical bulletin 'Fundamentals of Industrial Instrumentation' (Minneapolis-Honeywell Regulator Co., Philadelphia, Pa., U.S.A.) gives an adequate description for most purposes.

\section{Selection of control valve}

The most convenient choice for a valve to control the gas flow rate would be an electrically operated model working directly from the output of the controller. However, since such a valve was not available, it was necessary to use a pneumatic system consisting of an electro-pneumatic transducer and a pneumatic control valve. To achieve good automatic control it is essential that the control valve is the correct size. A wide range of suitable miniature pneumatic control valves with interchangeable trims are available from Research Controls Inc., Tulsa, Oklahoma, U.S.A. A single valve trim can be used for a wide range of requirements by suitably adjusting the composition of the oxygen-containing gas or the flow of inert gas into the fermentor. However, it is important to note that the higher the flow rate of gas through the fermentor the faster is the response to corrective action and the better the control. The trim used in the present work was chosen to allow a maximum gas flow of about $150 \mathrm{ml} . / \mathrm{min}$. with a $5 \mathrm{lb} . / \mathrm{in}^{2}{ }^{2}$ pressure drop across the valve.

\section{Equipment assembly}

The oxygen control equipment was fitted to a 2-litre 'Porton type' fermentor similar to that described by Elsworth, Meakin, Pirt \& Capell (1956). An unbaffled vortex aeration system was used, with a stirrer speed of $1440 \mathrm{rev} . / \mathrm{min}$. The inflowing gas mixture was introduced into the volume above the culture and the effluent gas was removed via the overflow weir. The temperature was automatically controlled to within $\pm \mathbf{0 \cdot 2 5 ^ { \circ }}$. The oxygen electrode was sterilized by ethylene oxide (Callow \& Pirt, 1956).

A schematic diagram of the control equipment is shown in Fig. 1; each component is specified in Table 1. Basically the equipment is a closed loop control system. The Mackereth electrode produces a current which is directly proportional to the dissolved oxygen tension in the culture, and which is measured by determining the potential drop across a resistance on a self-balancing potentiometric recorder. Depending on the deviation from the set-point, and on the rate of change of deviation, the 3-action control unit gives out a corrective signal varying between 1 and 5 $\mathrm{mA}(\mathrm{DC})$. The signal is fed into an electro-pneumatic transducer which converts it to a pneumatic signal varying proportionally between 3 and $15 \mathrm{lb}$./in. ${ }^{2}$ This signal, by acting on the diaphragm of the pneumatic control valve, varies the adjustment 
of the valve, which in turn varies the flow of the oxygen-containing gas proportionally to the initial corrective signal from the controller. The gas is then directed into a stream of inert gas flowing at a constant rate into the fermentor. The variation in flow of the oxygen-containing gas causes a corresponding variation in the oxygen partial pressure of the gas mixture above the culture, which is responsible for any necessary corrective action to the dissolved oxygen tension in the culture. The inert gas used was nitrogen, and the oxygen-containing gas was air.

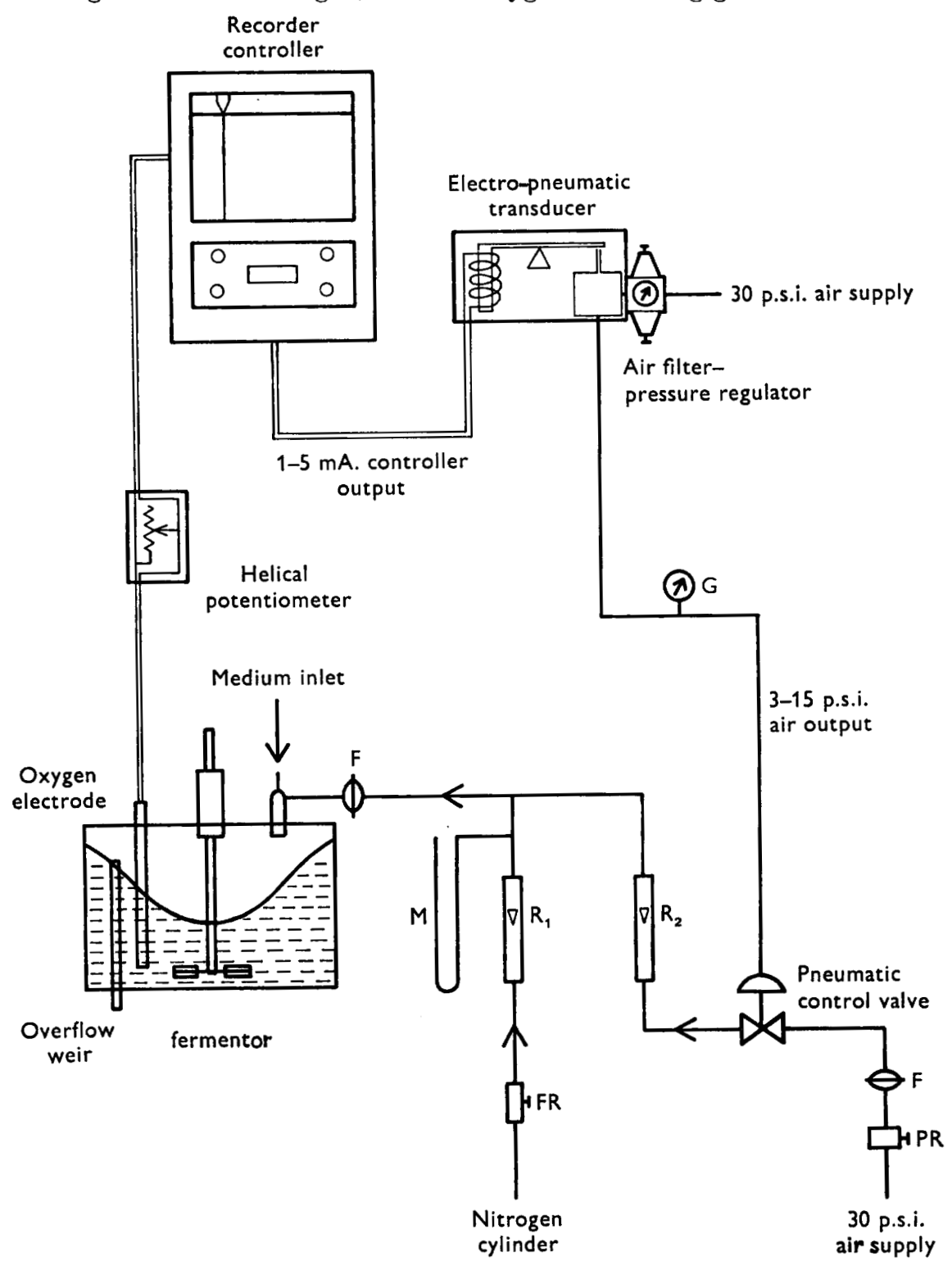

Fig. 1. Diagram of apparatus for the automatic control of dissolved oxygen tension in stirred microbial cultures. F, air filters; $M$, manometer; $R_{1}, R_{2}$, Rotameters; $G$, pressure gauge; FR, flow regulator; PR, pressure regulator. See Table 1 for specifications of components. 


\section{Table 1. Specifications of equipment for the automatic control of dissolved oxygen tension in stirred microbial cultures}

Electrode. 'Mackereth' laboratory oxygen electrode manufactured and supplied by Electronic Instruments Ltd., Richmond, Surrey. The electrode was modified for insertion through the fermentor head-plate by extending the length with a perspex sleeve. Price £31.

Helical potentiometer. 0-10 ohm, 10 turn 'Reliance' miniature helical potentiometer, type HEL 07-10, with 'Kilo' analogue dial and brake, type 462, manufactured and supplied by Reliance Controls Ltd., Walthamstow, London, E. 17. Price $£ 5$.

Recorder controller. 0-1 mV range 'Speedomax $H$ ' self-balancing potentiometric recorder with integrally mounted Series 60 3-action control unit, manufactured and supplied in the U.K. by Leeds and Northrup Ltd., Tysely, Birmingham. Recorder details: type, strip chart; chart speed, $1 \mathrm{in}$./hr; balance, 1.2 sec. nominal. Controller details : action, proportional + reset + rate; type, current adjusting; output, 1-5 mA at 15 V (DC). Price $\$ 330$.

Electro-pneumatic transducer. 'Fisher' electro-pneumatic transducer, type 543, with integrally mounted air filter-pressure regulator type 67 FR, manufactured and supplied by Fisher Governor Co. Ltd., Rochester, Kent. Transducer details: resistance, 2500 ohms; current input, 1-5 mA (DC); pneumatic output, 3-15 1b./in.2 Price $\$ 45$.

Pressure gauge (G, Fig. 1). 0-20 lb./in. ${ }^{2}$ Price $£ 2$.

Pneumatic control valve. $\frac{1}{4}$ in. air-to-open 'Minim' air operated miniature control valve; body, type 304 stainless steel with P4 trim ( $C_{v}$ max. (U.S.) 0.0006), manufactured by Research Controls Inc., Tulsa, Oklahoma, U.S.A., and supplied by G. A. Platon Ltd., Croydon, Surrey. Price e67; additional trim about £16.

Pressure regulator for culture air supply (PR, Fig. 1). 'Manostat' pressure regulator type 10, $\frac{1}{4}$ in. BSP, output range $2-25 \mathrm{lb}$./in. ${ }^{2}$, with high sensitivity adjustment, manufactured in the U.K. by John Watson and Smith Ltd., Leeds, Yorkshire, and supplied by G. A. Platon Ltd., Croydon, Surrey. Price \&7.

Flow regulator for culture $\mathrm{N}_{2}$ supply (FR, Fig. 1). 'Flostat minor' automatic flow regulator, MK. II AL, manufactured and supplied by G. A. Platon Ltd., Croydon, Surrey. Price \&8.

Gas flow indicators $\left(\mathbf{R}_{1}, \mathbf{R}_{2}\right.$, Fig. 1). Rotameters, ranges $R_{1}, 10-100 \mathrm{ml} . / \mathrm{min} ., \mathbf{R}_{2}, 5-150 \mathrm{ml}$./ min.; manufactured and supplied by the Rotameter Manufacturing Co. Ltd., Croydon, Surrey. Price £16.

Total cost $£ 511$.

\section{Calibration}

The Mackereth electrode was calibrated in sterile medium before inoculation. The system was allowed to equilibrate with an air flow rate of about $200 \mathrm{ml}$./min. until a steady recorder reading was obtained. With a freshly prepared electrode this usually took about $24 \mathrm{hr}$. The measuring range was then set by adjusting the helical potentiometer so that the recorder indicated a particular value between 0.95 and $0.98 \mathrm{mV}$. The brake on the potentiometer dial was then locked. The linearity of the electrode was checked by substituting at least two other gases of known composition before determining zero dissolved oxygen tension with oxygen-free nitrogen. After correction for barometric pressure, back pressure, and saturated water vapour pressure, the partial pressure $(\mathrm{mm}$. $\mathrm{Hg}$ ) of oxygen in the gas phase for each gas mixture was plotted against the corresponding recorder readings $(\mathrm{mV})$. Since the partial pressure of oxygen in the gas phase was in equilibrium with the oxygen dissolved in the medium, the dissolved oxygen tension in the medium could thus be read directly from the graph. The current produced by the electrode at any time was calculated by dividing the relevant potential drop $(\mathrm{mV})$ by the resistance, the latter being read directly from the dial of the helical potentiometer.

The measuring system was always re-calibrated after an experiment, to check for 
drift. Before re-calibration the culture was discarded, the fermentor thoroughly flushed with sterile medium, and the equipment left overnight to equilibrate.

The calibration procedure described is for a measuring range of $0-100 \%$ air saturation (or approximately 0-155 mm. Hg dissolved oxygen tension). By substituting gases of appropriate composition the recorder can be calibrated over any desired range. The following gases have been used for calibration (obtained from British Oxygen Co. Ltd., North Wembley, Middlesex): 10, 5, 2, and 1\% (v/v) oxygen in nitrogen; oxygen-free nitrogen.

\section{Tuning of the controller}

Once the equipment had been installed, all that was necessary to set it in operation was to select the set-point, fix the nitrogen flow rate, and 'tune' the controller by adjusting three dials which determined the width of the proportional band and the degree to which reset and rate actions were required. The controller was tuned according to the directions in the comprehensive explanatory booklet supplied by the manufacturers. After becoming familiar with the equipment no difficulty was experienced in the tuning process. When the conditions in the process were to be drastically changed from those under which the controller was tuned, a further adjustment was often necessary to ensure good control. Table 2 shows typical proportional band, rate and reset adjustments used under various experimental conditions.

\section{RESULTS}

The equipment has been used in a series of experiments involving both batch and continuous culture of a pseudomonad where the dissolved oxygen tension has been controlled at various values down to $\mathbf{0 . 2 6} \mathrm{mm}$. Hg. During this work the system has shown a high degree of stability over periods up to 5 weeks, and proved capable of controlling the dissolved oxygen tension to within very fine tolerances. The experiments are summarized in Table 2.

Fig. $2 a$ and $b$ show sections of typical charts for dissolved oxygen tension controlled at $30 \mathrm{~mm}$. $\mathrm{Hg}$ and $1 \mathrm{~mm}$. $\mathrm{Hg}$, respectively, during the continuous culture of the pseudomonad grown on the hydrocarbon decane. Fig. $2 a$ is the control trace obtained when the culture was grown under nitrogen-limited conditions, at a concentration of organisms equiv. $2 \cdot 22 \mathrm{mg}$. dry wt/ml. Fig. $2 b$ shows the trace when the dissolved oxygen tension was growth-limiting, the corresponding concentration of organisms being equiv. $1.44 \mathrm{mg}$. dry wt/ml. It can be seen that the control traces were virtually straight lines even when the dissolved oxygen tension was the growthlimiting factor.

Fig. $3 a$ and $b$ show the response of the controller to a disturbance artificially induced by switching off the stirrer in the fermentor. The electrode registered a decrease in dissolved oxygen tension followed by an increase when the stirrer was switched on. In the figure the arrow denotes the point at which the true control action began. It can be seen that in returning the dissolved oxygen tension to the set-point no overshoot or hunting occurred.

Fig. 4 shows a chart for dissolved oxygen tension controlled at $30 \mathrm{~mm}$. Hg during the batch culture of the same pseudomonad with glucose as the carbon source. Over the 9-hr period shown in the figure, the concentration of bacteria and the oxygen 
Table 2. Summary of experiments with the automatic equipment for controlling dissolved oxygen tension

Cultural details refer to growth of a pseudomonad on defined medium at $30^{\circ}$. Foam was controlled throughout all experiments by the automatic addition at 2-hr intervals of about 0.1 ml. of polyglycol P-2000 (Dow Chemical Co., U.K., Ltd., London, W. 1). In all cases the inert gas was $\mathrm{N}_{2}$ at a flow rate of about $90 \mathrm{ml}$./min. The oxygen-containing gas was air.

\begin{tabular}{|c|c|c|c|c|c|c|c|c|}
\hline & & & & $\begin{array}{c}\text { Effi- } \\
\text { ciency }\end{array}$ & Con & troller adj & nent & \\
\hline $\begin{array}{c}\text { Con- } \\
\text { trolled } \\
\text { dissolved } \\
\mathrm{O}_{2} \\
\text { tension } \\
(\mathrm{mm} . \mathrm{Hg})\end{array}$ & $\begin{array}{l}\text { Growth- } \\
\text { limiting } \\
\text { factor }\end{array}$ & $\begin{array}{c}\text { state } \\
\text { bacterial } \\
\text { concn. } \\
\text { (equiv. } \\
\text { mg. dry } \\
\text { wt./ml.) }\end{array}$ & $\begin{array}{l}\text { Air } \\
\text { flow } \\
\text { (ml./ } \\
\text { min.) }\end{array}$ & $\begin{array}{c}\text { oxygen } \\
\text { utili- } \\
\text { zation } \\
\text { ( } \% \text { of } \\
\mathrm{O}_{2} \\
\text { input) }\end{array}$ & $\begin{array}{c}\text { Pro- } \\
\text { portional } \\
\text { band } \\
\text { ( } \% \\
\text { recorder } \\
\text { scale) }\end{array}$ & $\begin{array}{c}\text { Reset } \\
\text { (repeats/ } \\
\text { min.) }\end{array}$ & $\begin{array}{c}\text { Rate } \\
\text { time } \\
\text { (min.) }\end{array}$ & $\begin{array}{c}\text { Fig. no. } \\
\text { of } \\
\text { typical } \\
\text { chart }\end{array}$ \\
\hline \multicolumn{9}{|c|}{ Continuous culture, dilution rate $0 \cdot 1 \mathrm{hr}^{-1}$; $\mathrm{C}$ source decane } \\
\hline $30 \cdot 0$ & $\mathbf{N}$ source & $\mathbf{2 \cdot 2 2}$ & 117 & 57 & 25 & 0.06 & $0 \cdot 04$ & $2 a, 3 a$ \\
\hline $14 \cdot 0$ & $\mathbf{N}$ source & $2 \cdot 06$ & 90 & 70 & 25 & 0.06 & $0 \cdot 04$ & - \\
\hline $5 \cdot 7$ & $\mathbf{N}$ source & $\mathbf{2} \cdot 18$ & 71 & 80 & 19 & 0.06 & 0.025 & - \\
\hline $3 \cdot 0$ & N source & $2 \cdot 12$ & 53 & 84 & 19 & $0 \cdot 06$ & $0 \cdot 025$ & $3 b$ \\
\hline $1 \cdot 0$ & $\begin{array}{l}\text { Dissolved } \\
\mathrm{O}_{2} \text { tension }\end{array}$ & $1 \cdot 44$ & 19 & 86 & 19 & 0.06 & 0.025 & $2 b$ \\
\hline \multicolumn{9}{|c|}{ Batch culture, 9-hr duration; C source glucose } \\
\hline $30 \cdot 0$ & None I & $\begin{array}{l}\text { Increase } \\
\text { from } 0 \cdot 64 \\
\text { to } 3 \cdot 46\end{array}$ & $\begin{array}{l}\text { Increase } \\
\text { from } 55 \\
\text { to } 160\end{array}$ & - & 30 & $0 \cdot 3$ & $0 \cdot 0$ & 4 \\
\hline
\end{tabular}

uptake increased about fivefold. It can be seen that the controller satisfactorily maintained the dissolved oxygen tension at the set-point despite the large load changes encountered. It is important to note that for use with a batch culture, the controller had to be tuned to cope with the very large load changes which occur towards the end of exponential growth, i.e. a high reset value was required. However, in the initial stages of the batch culture where load changes were relatively smaller and slower, the high reset rendered the system ultrasensitive, thus giving rise to cycling. This effect can be seen in Fig. 4. The deviations in the initial stages would be of little consequence for most practical purposes.

With the exception of the batch culture (Fig. 4), all work reported in this paper was done with the electrode sheathed with a 0.001 in. thick membrane made from fluoroethylenepropylene polymer (FEP; Du Pont Co., U.K., Ltd). The FEP membrane was used primarily because of its resistance to hydrocarbons (MacLennan \& Pirt, 1965). However, the same control precision was obtained when using the standard polythene membrane (0.0025 in. thickness) supplied with the electrode, in systems not involving hydrocarbons. The batch culture trace shown in Fig. 4 was obtained with the standard polythene membrane.

One defect in all membrane electrodes is their sensitivity to temperature fluctuation. Fig. 5 shows a typical recording of the dissolved oxygen tension in sterile medium at air saturation $\left(155 \mathrm{~mm} . \mathrm{Hg}, \mathrm{O}_{2}\right.$ ) when using an FEP membrane. The cycle in the trace is entirely due to temperature fluctuations resulting from automatic temperature control $( \pm \mathbf{0 \cdot 2 5})$. The temperature sensitivity for the FEP membrane was thus about $2 \%$ of the reading/degree, and not $5 \%$ as estimated previously 


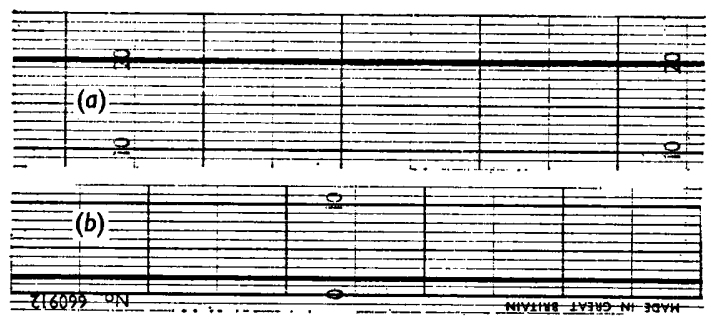

Fig. 2. Sections of typical charts showing controlled dissolved oxygen tension during a continuous culture of a pseudomonad on a defined medium (C source, decane). $(a)$ Controlled at $30 \mathrm{~mm}$. Hg (growth limited by $\mathrm{N}$ source); $(b)$ controlled at $\mathbf{1 ~ m m}$. $\mathbf{H g}$ (growth limited by dissolved oxygen tension). The heavy vertical lines represent intervals of $1 \mathrm{hr}$. Each small horizontal division corresponds to $1.6 \mathrm{~mm}$. $\mathrm{Hg}$ dissolved oxygen tension.
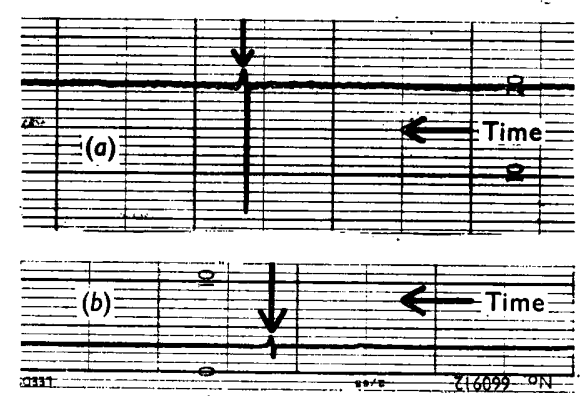

Fig. 3. Charts showing the response of the controller to an artificially induced interference in the dissolved oxygen tension caused by switching off the stirrer during a continuous culture of a pseudomonad on a defined medium ( $C$ source, decane). The vertical arrows indicate the point at which true control action began. (a) Controlled at $30 \mathrm{~mm}$. $\mathrm{Hg}$ dissolved $\mathrm{O}_{2}$ tension; $(b)$ controlled at $3 \mathrm{~mm}$. Hg. The heavy vertical lines represent intervals of $1 \mathrm{hr}$. Each small horizontal division corresponds to $1.6 \mathrm{~mm}$. $\mathrm{Hg}$ dissolved oxygen tension.

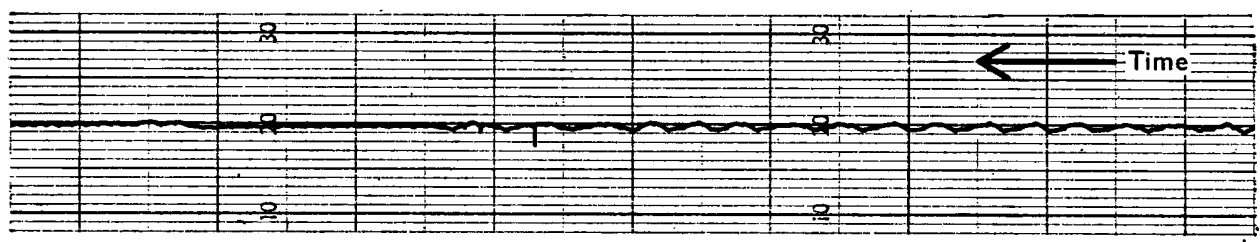

Fig. 4. Chart showing dissolved oxygen tension controlled at $30 \mathrm{~mm}$. $\mathrm{Hg}$ during a batch culture of a pseudomonad on a defined medium (C source, glucose). Over the 9-hr period shown the concentration of bacteria and the oxygen uptake increased about fivefold. The Mackereth electrode was sheathed with the standard polythene membrane ( 0.0025 in. thickness). The heavy vertical lines represent intervals of $1 \mathrm{hr}$. Each small division corresponds to $1 \cdot 6 \mathrm{~mm}$. $\mathrm{Hg}$ dissolved oxygen tension. 


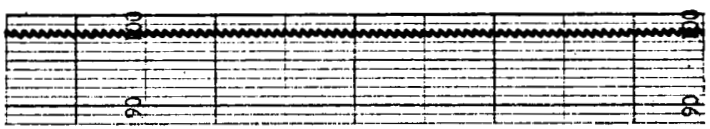

Fig. 5. Chart showing the effect of temperature fluctuation on the output from the Mackereth electrode (FEP membrane, 0.001 in. thick) measured in sterile medium at $30^{\circ}$ and at a dissolved oxygen tension of $155 \mathrm{~mm}$. Hg. The cycle in the trace is due to fluctuations of $\pm \mathbf{0 . 2 5}$ in the automatic temperature control. The temperature coefficient of the electrode reading is about $2 \% /$ degree. The heavy vertical lines represent intervals of $1 \mathrm{hr}$. Each small horizontal division corresponds to $1.6 \mathrm{~mm}$. $\mathrm{Hg}$ dissolved oxygen tension.

(MacLennan \& Pirt, 1965). The lower the dissolved oxygen tension the less appreciable the temperature effect became. In Figs. $2 a$ and $3 a\left(30 \mathrm{~mm} . \mathrm{Hg}, \mathrm{O}_{2}\right)$ the effect is barely visible, and in Figs. $2 b\left(1 \mathrm{~mm} . \mathrm{Hg}, \mathrm{O}_{2}\right)$ and $3 b\left(3 \mathrm{~mm} . \mathrm{Hg}, \mathrm{O}_{2}\right)$ it cannot be seen at all.

Contrary to expectation the periodic addition of antifoam (2-hr intervals) as used throughout this work produced no change in the dissolved oxygen tension in the culture. It is significant that the rate of antifoam addition was such as to prevent the formation of foam rather than to control the foam level.

An important feature of this automatic control system, particularly from the industrial point of view, was the high efficiency of oxygen utilization. From Table 2 it can be seen that the efficiency ranged from $57 \%$ at a controlled dissolved oxygen tension of $30 \mathrm{~mm} . \mathrm{Hg}$ to $86 \%$ at $1 \mathrm{~mm}$. $\mathrm{Hg}$.

The stability of the electrode can be assessed from Fig. 6, which shows calibration curves for the electrode both before and after a 5-week continuous culture run in which the sole carbon source was decane. It can be seen that the output from the

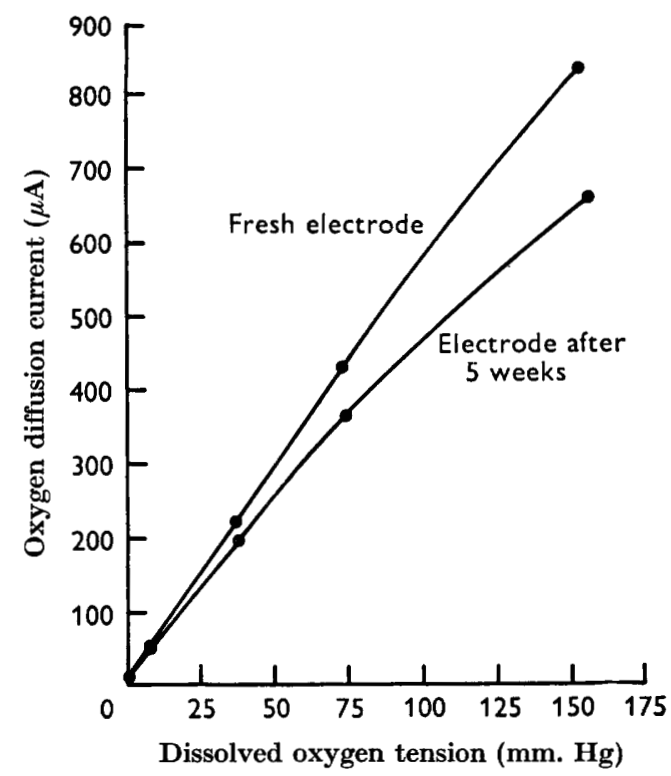

Fig. 6. Calibration curves for a Mackereth electrode (FEP membrane, 0.001 in. thick) before and after a 5-week continuous culture of a pseudomonad on a defined medium (C source, decane). The dissolved oxygen tension was controlled at various values between 30 and $1 \mathrm{~mm}$. Hg. 
fresh electrode tended to show a slight change from linearity at high dissolved oxygen tensions (above about $100 \mathrm{~mm}$. $\mathrm{Hg}$ ). At the end of the run the output from the electrode had decreased and showed a more marked change from linearity at high dissolved oxygen tensions. However, in the latter curve the deviation from linearity was negligible below about $75 \mathrm{~mm} . \mathrm{Hg}, \mathrm{O}_{2}$. It is in this region that the most interesting effects of dissolved oxygen tension on the behaviour of microbial cultures are to be expected. In this region the electrode sensitivity decreased from 5.82 to $5.02 \mu \mathrm{A} / \mathrm{mm}$. $\mathrm{Hg}, \mathrm{O}_{2}$, over the 5-week period. The sensitivity could thus be expressed as the mean of these two values, $5 \cdot 42 \mu \mathrm{A} / \mathrm{mm} . \mathrm{Hg}, \mathrm{O}_{2}$, with a maximum deviation of $7.5 \%$. Alternatively, the average decrease in sensitivity was $0.4 \%$ of the initial value/day. The residual current remained at the same value, $9 \mu \mathrm{A}$, throughout the period. All of the work described so far was done with an early model of the Mackereth electrode (model A 15 A). More recently, however, a better performance has been obtained with a later model of the electrode (model A 15000, 0.001 in. thick FEP membrane), during the continuous culture of the pseudomonad in which the sole carbon source was glucose. In this work the recorder was calibrated over a range $0-39 \mathrm{~mm}$. $\mathrm{Hg}$ dissolved oxygen tension, and the equipment was used to control the dissolved oxygen tension at various values between $0 \cdot 26$ and $30 \mathrm{~mm}$. Hg. Over the 5-week period the mean sensitivity was $7 \cdot 67 \mu \mathrm{A} / \mathrm{mm} . \mathrm{Hg}, \mathrm{O}_{2}$, with a maximum deviation of $3.6 \%$ (average decrease in sensitivity, $0.2 \%$ of the initial value/day). The residual current remained constant at $2 \cdot 9 \mu \mathrm{A}$. Thus within the range $0-75 \mathrm{~mm} . \mathrm{Hg}, \mathrm{O}_{2}$, both models of the electrode showed a sufficiently high degree of stability to ensure accurate and reliable automatic control of dissolved oxygen tension over periods of at least 5 weeks.

\section{DISCUSSION}

The equipment described in this paper provides, for the first time, a precise, reliable and versatile system for the automatic control of dissolved oxygen tension in microbial cultures. The equipment was selected with plant operation as well as laboratory use in mind. All the components used are commercially available and are of first quality, precision industrial grade. Such equipment is expensive, but by far the most economical in the long run because of its stability, reliability and trouble-free operation, factors often lacking in cheaper instruments. The equipment is not limited to the use of the Mackereth electrode, but can be used with any oxygen electrode whose output can be converted to millivolts. The extremely low range of the recorder is a great asset in this respect.

The straight line control traces obtained in this work could possibly have been the result of such an extremely sluggish and insensitive electrode response that the traces did not reflect actual changes occurring in the dissolved oxygen tension. This, however, is considered most unlikely on the grounds of the rapid response to artificially induced disturbances shown in Fig. $3 a$ and $b$, and also because small changes in controlled dissolved oxygen tension (for example, from 1 to $\mathbf{0 . 2 6} \mathrm{mm}$. $\mathrm{Hg}$ ) have produced significant and reproducible changes in culture mass.

It is important to note that the decrease in sensitivity of the FEP-sheathed electrodes was the largest observed for any Mackereth electrode used in these laboratories. The best performance was obtained with an electrode with a polythene membrane ( 0.0025 in. thick), a negligible decrease being observed over a 
period of 8 weeks continuous use followed by a $10 \%$ decrease in the following week (D. E. F. Harrison, personal communication). The higher current output from the FEP-sheathed electrodes would probably be responsible for the increased deviation from linearity at high dissolved oxygen tensions and the change in long-term stability observed. In the present work the thin FEP membrane was used specifically to decrease the response time of the electrode (Mackereth, 1964), since it was initially thought that the slow response of the electrode would be the critical factor in obtaining precise automatic control. Subsequent work, however, showed that good control could be achieved when using the thicker polythene membrane (see Fig. 4). Hence a thicker FEP membrane could be used to decrease the current output and possibly improve the long-term stability.

One criticism of the partial pressure method of controlling dissolved oxygen tension in continuous cultures was that it would be difficult to determine the oxygen uptake of the culture because of variation in oxygen input. In our experience, the air input has been found to remain remarkably constant once a steady state has been attained, and no difficulty has been encountered in determining oxygen uptake rates, irrespective of whether the culture was grown at excess, critical or limiting dissolved oxygen tensions. This might be due to the stabilizing effect of controlled dissolved oxygen tension, which would tend to damp rather than amplify potential oscillations in the culture metabolism.

At the present time the extent to which rate action is necessary in controlling dissolved oxygen tension in fermentation systems has not been fully investigated. It has been included in the equipment to provide maximum versatility in coping with any situation which may arise during microbial cultivation, and has been found necessary in all circumstances so far examined.

Although no experimental evidence of control below $0.26 \mathrm{~mm} . \mathrm{Hg}, \mathrm{O}_{2}$, is available, there is no reason why it should not be feasible, since the Mackereth electrode produces a linear response down to zero dissolved oxygen tension, and the control equipment has ample potential to cope with sensitive and unstable systems. It is important to note that the equipment described is capable of controlling the dissolved oxygen tension well below the levels which can be tolerated by such 'strict anaerobes' as Clostridium tetani (2 $\mathrm{mm}$. $\mathrm{Hg}, \mathrm{O}_{2}$; Gordon, Holman \& McLeod, 1953).

The authors thank both the Science Research Council and Imperial Chemical Industries Ltd. for financial support for this work.

\section{REFERENCES}

ArNold, B. H. \& SteEL, R. (1958). Oxygen supply and demand in aerobic fermentations. In Biochemical Engineering. Ed. by R. Steel, p. 149. London: Heywood and Co. Ltd.

Callow, D. S. \& Pirt, S. J. (1956). Automatic control of $\mathrm{pH}$ value in cultures of microorganisms. J. gen. Microbiol. 14, 661.

Elsworth, R., Meakin, L. R. P., Pirt, S. J. \& Capell, G. H. (1956). A two litre scale continuous culture apparatus for micro-organisms. J. appl. Bact. 19, 264.

Gordon, J., Holman, R. A. \& McLeod, J. W. (1953). Further observations on the production of hydrogen peroxide by anaerobic bacteria. J. Path. Bact. 66, 527.

Harrison, D. E. F. \& Pirt, S. J. (1965). Oxygen tension and glucose metabolism of Klebsiella aerogenes. J. gen. Microbiol. 41, ix.

Herbert, D., Phipps, P. J. \& Tempest, D. W. (1965). The chemostat: design and instrumentation. Lab. Pract. 14, 1150. 
Kinsey, D. W. \& BotтomLey, R. A. (1963). Improved electrode system for determination of oxygen tension in industrial applications. J. Inst. Brerv. 69, 164.

LENGYEL, Z. L. \& NYIRI, L. (1965). An automatic aeration control system for biosynthetic processes. Biotechnology and Bioengineering 7, 91.

MACKERETH, F. J. H. (1964). An improved galvanic cell for determination of oxygen concentration in fluids. $J$. scient. Instrum. $41,38$.

MacLennan, D. G. \& Pirt, S. J. (1965). A new membrane for electrodes measuring dissolved oxygen tension. Chemy Ind. p. 1973.

Porter, R. W. \& Considine, D. M. (1950). Process control. In Chemical Engineers' Handbook, 3rd ed. Ed. by J. H. Perry, p. 1263. New York: McGraw-Hill Book Co. Inc. Siegell, S. D. \& Gaden, E. L. (1962). Automatic control of dissolved oxygen levels in fermentations. Biotechnology and Bioengineering 4, 345. 